\title{
Optics
}

\section{Overall Optical Design of LED Surgical Luminaires of Variable Light Spot}

\author{
Shikang Zhou*, Chungen Chen \\ Email address: \\ 2447441104@qq.com (Shikang Zhou) \\ ${ }^{*}$ Corresponding author
}

Shanghai Sansi Electronic Engineering Technology Co., Ltd, Shanghai, China

To cite this article:

Shikang Zhou, Chungen Chen. Overall Optical Design of LED Surgical Luminaires of Variable Light Spot. Optics. Vol. 8, No. 1, 2019 , pp. 1-6. doi: $10.11648 /$ j.optics.20190801.11

Received: December 17, 2018; Accepted: January 22, 2019; Published: February 21, 2019

\begin{abstract}
The surgical luminaires play a very important role in surgical operation and diagnostics. Its overall optical design is the most important component in the design of the surgical luminaires. The wide variable range of the spot size makes the luminaires adaptable to multiple occasions, and the variability is an important indicator for high-grade surgical luminaires. However, it is difficult to keep the uniformity of illumination when the size of the light field changes. One of the reasons why there are few new surgical lamps with wide range of spot size variation in the market is the lack of theoretical analysis. In this paper, we analyze the relationship between the optics of surgical luminaries and the variable light spot by examining a hypothetical surgical lamp composed of 7 LEDs, 6 of which can move radially to create the total spot variable. An improved multivariate Gaussian function will be used to simulate the variable illumination field. Several important optical parameters describing the light field in IEC standard are carefully selected as the independent variables of the Gaussian function. By adding seven improved Gaussian functions of single-spot, a mathematical function of all-spot illumination distribution is established. By this way, it is easy to find in what manner the illumination distribution of surgical luminaires varies with independent variables under the condition of meeting the requirements of international standards. From the calculated results, the important parameters for overall optical design are determined, such as the illumination distribution, the amount of spot movement, the maximum variation range of the light field and the uniformity of illumination. The graphical representation of the results are also given in this paper. The original calculations provided in this article can be used in overall optical design of surgical luminaires with any range of light field change.
\end{abstract}

Keywords: Surgical Luminaire, Shadow-Less Lamp, Variable Light Spot, Optical Design, Illumination, LED

\section{Optical Requirements for Surgical Luminaires}

According to statistics, more than 50 million surgeries are performed each year in the world. Surgical luminaires are the most important equipment in surgical operating room. The publication [1] revised by the International Electro-technical Commission (IEC) in 2009 is the most authoritative standard for surgical luminaires. As a member of IEC, the Chinese standard of surgical luminaires [2] is basically the same as that of IEC. The design of surgical luminaires covers a wide range of areas, including optics, machinery, electricity, control, appearance, safety and so on. This paper will not give a comprehensive introduction to the design of surgical luminaires, but focus specifically on some of the most important optical problems that must be considered in the overall design of variable spot surgical lamps.

According to the IEC standard, the following parameters are used to describe the light field of a surgical lamp:

Light field center LFC: usually the point of maximum illumination in the light field.

Center illuminance Ec: the illuminance at LFC

Light field diameter $D_{10}$ : diameter of a circle around LFC where the illuminate reaches $10 \%$ of Ec. See Figure 1

The distribution of light field parameter $D_{50}$ : diameter of a circle around LFC, the illumination on the circle is $50 \%$ of $\mathrm{E}_{\mathrm{C}}$.

Depth of illumination: the depth of the region below the emitting surface, of which the illumination is at least $60 \%$ of that of $\mathrm{E}_{\mathrm{C}}$.

As article [3] described, IEC standard is the most important document for the design of surgical lamp. 
However the requirement of IEC standard for the distribution of surgical lamp light field is very simple. That is the minimum diameter $\mathrm{D}_{50}$ needs to be at least $50 \%$ of $\mathrm{D}_{10}$. This requirement can be expressed as (1) and (2):

$$
\begin{gathered}
c=D_{50} / D_{10} \\
c \geq 0.5
\end{gathered}
$$

In fact, (1) and can be visually understood as requiring a certain degree of "fullness". The larger the c is, the "fuller" the shape is, and the more uniform the illumination will be. Thus we will be referring to the shape parameter "c" as "fullness" in this paper.

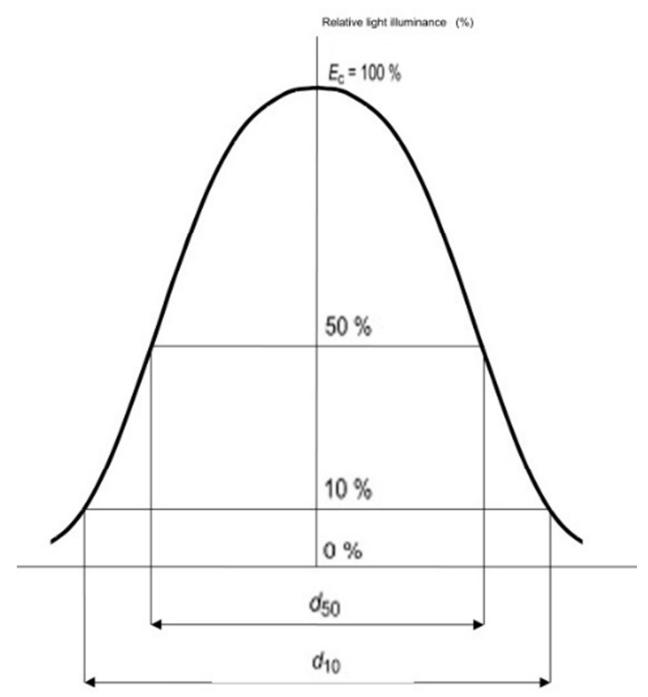

Figure 1. Light distribution of surgical field, (quoted from [1] p22).

\section{LED Surgical Luminaires}

Traditional surgical luminaries commonly use halogen lamps as light source. Halogen lamps have multiple problems, including short life span, large light volume and slow start up. The most critical flaw is that it generates a large amount of heat and can result in wound overheating. Ultraviolet radiation from halogen lamps can even cause skin cancer. Reference [4] has carried out a large number of investigations in 39 countries, proving that the use of halogen lamps can be harmful. Reference [5] demonstrates that higher temperature can cause an increase in particulate matter during surgeries.

In contrast, the surgical luminaries with a LED light source have many unique advantages [6]. LED as the cold light source, it will not produce any infrared and ultraviolet radiation. It will not cause wound temperature to rise and accelerate blood coagulation, which is conducive to the recovery of wound after surgery. Surgeons can easily adjust the brightness and color temperature according to different tissues. LED luminescence is directional, and thus is it easy to distribute the light in a controlled fashion, restricting it to only areas which light is required. Several kinds of LED light sources can be mixed to improve lighting [7]. On top of this, LEDs also have a longer life time, start up instantly, and can use low voltage DC batteries. It has become an industry-wide trend for LED lamps to replace their halogen counterparts. A large number of LED surgical luminaries have appeared in the market, (ref [8-12]), and there are a lot of publications on LED surgical lamp, such as the optical characteristics of the lamp [14], reflection type lamp [15], the reliability of the lamps [16] and the stability of power supply [17].

As an example of surgical luminaires of variable light spot, the lamp in this paper consists of one central LED module and six LED modules arranged in a circle around it. Their seven light spots synthesize total light field at a distance of $1 \mathrm{~m}$ from the LED. Appropriate mechanical design [13] enables six peripheral light fields to move synchronously along the radial direction to adjust the total light field.

\section{Surgical Luminaires of Variable Light Spot}

From a practical perspective, variable diameter of the surgical lamps are obviously more suitable for different surgical needs than fixed spot lamps in a surgical operating room. However, most of the surgical lamps on the market do not have a variable diameter, and the ones that do have poor illumination uniformity. The reason is that designers encountered difficulties, such as how to describe the lighting distribution and illumination uniformity mathematically, and how to meet the requirements of IEC standard in the adjustment process. As a result there is hardly any research in the area of lamps with adjustable light field. The method [18] proposed in this paper attempts to address and solve this problem. We successfully used the method of simulation calculation with a modified Gaussian function to study the regularity of spot variety. Then obtained the most important data in the overall design include maximum range of diameter change, the most suitable single spot shape and the highest total spot uniformity of the lamp.

It should be pointed out here that since the IEC standard [1] was established before the emergence of a large number of variable spot surgical lamps, which have a bell shaped illumination intensity distribution, there is no explicit requirement for uniformity in the standard, but only the requirement of "fullness". This has not proven to be a problem in early years [3]. But the surgical lamps with variable spot often have multi-module, and their spot is not always "bell-shaped" in the adjustment. On the other hand, the uniformity of illumination is an obvious requirement in surgery. It can be said that how to maintain uniformity in the process of changing the spot is a new problem brought by the new surgical lamps

\section{Modified Gaussian Function to Simulate the Illumination Distribution of Surgical Lamp}

The Gaussian function [19] has important applications in natural sciences, Social Sciences, mathematics and 
engineering. In the field of optics, the intensity distribution of a laser beam with parallel beam shape has proven to be a Gaussian function [20]. Among the issues we are discussing, the beam angle of surgical lamp is very small, it has a near parallel light beam shape, and so the intensity distribution could and should also be described using a onedimensional Gaussian function [21]

$$
y=\exp \left(-\frac{1}{\sqrt{2}}\left(\frac{r}{w}\right)^{2}\right)
$$

Here $r$ is the distance from the origin of coordinate, $w$ is the half width of the function peak. In order to describe the illumination distribution of surgical lamp better, the function (3) should be modified. To do this, add parameters $\mathrm{d}$ and $\mathrm{a}(\mathrm{c})$, then change (3) to (4):

$$
y=\exp \left(-d\left(\frac{|r|}{w}\right)^{a(c)}\right)
$$

The fixed exponent 2 in formula (3) was changed to the function a (c), then the relationship between illumination $y$ and shape parameter $\mathrm{c}$ can be described. And $\mathrm{d}$ is a parameter describing the width of the spot.

You can find an interesting but important and useful property of (4) that when $\mathrm{w}$ is fixed, the curves of different $\mathrm{a}(\mathrm{c})$ will intersect at the point of $\mathrm{r}=\mathrm{w}$ and the height of the intersection is $y=e-d$. Then $d$ can be used as a parameter to control the height of intersection points. This property can be seen more clearly in Figure 2. This is why we add parameter d into (4).

On the other hand, as can be seen from Figure $1, D_{10}$ is the size of the spot, so make the intersection point is 0.1 high:

$$
0.1=\exp (-d)
$$

Solving the equation, we get

$$
d=2.30259
$$

So function can be turn into

$$
y=\exp \left(-2.30259\left(\frac{|r|}{w}\right)^{a(c)}\right)
$$

Then the following equation is solved by the definition of $D_{10}$

$$
0.1=\exp \left(-2.30259\left(\frac{|r|}{D_{10} / 2}\right)^{a(c)}\right)
$$

Get (6):

$$
D_{10}=\frac{2 \mathrm{r}}{\exp \left(-2.58592 * 10^{(-12)} / a(c)\right)}
$$

Similarly, $D_{50}$ can be obtained as (7):

$$
D_{50}=\frac{2 \mathrm{r}}{\exp (-1.20054 / \mathrm{a}(\mathrm{c}))}
$$

From simultaneous equation (1), (6) and (7), a(c) can be get as

$$
a(c)=-\frac{\ln (\ln (2) / \ln (10)}{\ln (c)}=\frac{1.200545}{\ln (c)}
$$

The expression of illumination distribution can be obtained as follows

$$
f_{0}(r, b, c)=\exp \left(-2.30259\left(\frac{|r|}{w}\right)^{\frac{1.200545}{\ln (c)}}\right)
$$

With this multivariate function (9), we can mathematically describe the illumination distribution of surgical lamp with the spot radius $w$ and the saturation parameter c conveniently. Graphics of the functions can be easily drawn by using mathematical software Maple or Mathlab. Figure 2 shows the curves of illumination function $f_{0}(r, b, c)$ varying from $\mathrm{c}=0.5$ to 0.9 with a fixed single spot radius $\mathrm{w}=100 \mathrm{~mm}$. The curves intersect at $\mathrm{r}=100$. This kind of figure can easily describe various illumination distributions when the spot is $D_{10}$, and it helps us complete our analysis in this paper.

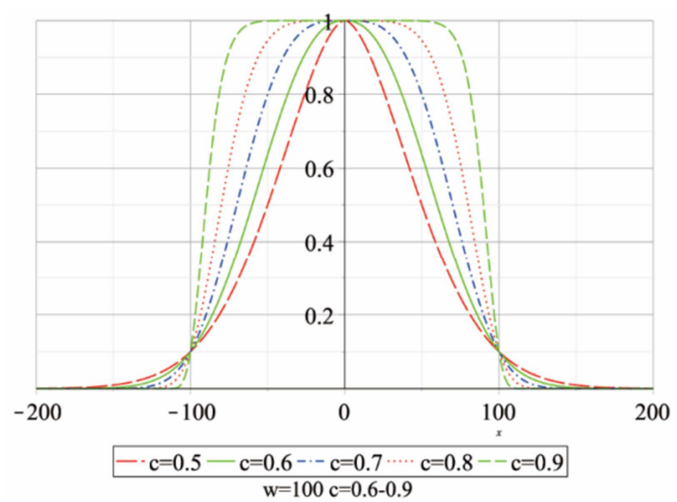

Figure 2. Illumination distribution function of single light spot.

The illumination area is composed of seven light beams, which are described by seven two-dimensional Gaussian functions [22]. The seven beams are shown in Figure 3

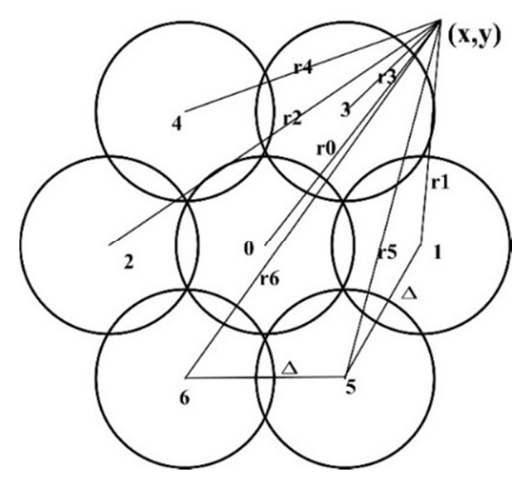

Figure 3. Schematic diagram of 7 light spots. 
The coordinate origin of $\mathrm{x}$ and $\mathrm{y}$ is at the center of the beam number 0 , and the other six beams are symmetrically distributed. All the distance between two adjacent centers are $\Delta$. The radii of these seven circles can be expressed by (10).

$$
\left\{\begin{array}{l}
r_{0}=\sqrt{x^{2}+y^{2}} \\
r_{1}=\sqrt{(x-\Delta)^{2}+y^{2}} \\
r_{2}=\sqrt{(x+\Delta)^{2}+y^{2}} \\
r_{3}=\sqrt{\left(x-\frac{1}{2} \Delta\right)^{2}+\left(y-\frac{\sqrt{3}}{2} \Delta\right)^{2}} \\
r_{4}=\sqrt{\left(x+\frac{1}{2} \Delta\right)^{2}+\left(y-\frac{\sqrt{3}}{2} \Delta\right)^{2}} \\
r_{5}=\sqrt{\left(x-\frac{1}{2} \Delta\right)^{2}+\left(y+\frac{\sqrt{3}}{2} \Delta\right)^{2}} \\
r_{6}=\sqrt{\left(x+\frac{1}{2} \Delta\right)^{2}+\left(y+\frac{\sqrt{3}}{2} \Delta\right)^{2}}
\end{array}\right.
$$

Expression (9) is the illumination distribution function of beam number 0 . The all seven functions with five independent variables then can be written as (11) $(\mathrm{i}=0 \ldots 6)$.

$$
\begin{aligned}
& f i(x, y, w, c, \Delta)= \\
& \exp \left(-2.30259\left(\frac{r_{i}(x, y, \Delta)}{w}\right)^{\frac{1.200545}{\ln (c)}}\right)
\end{aligned}
$$

According to the superposition principle of incoherent light, the total spot distribution with five independent variables is simply the sum of seven Gaussian functions:

$$
F(x, y, w, c, \Delta)=f_{0}+f_{1}+f_{2}+f_{3}+f_{4}+f_{5}+f_{6}
$$

On the special cross section passing through the center of the spot $(y=0)$, the illumination distribution of three spots (13) can be simplified as a function of four independent variables

$$
\left\{\begin{array}{l}
f x_{0}(x, w, c, \Delta)=\exp \left(-2.30259\left(\frac{|x|}{w}\right)^{\frac{1.200545}{\ln (c)}}\right) \\
f x_{1}(x, w, c, \Delta)=\exp \left(-2.30259\left(\frac{|x-\Delta|}{w}\right)^{\frac{1.200545}{\ln (c)}}\right) \\
f x_{2}(x, w, c, \Delta)=\exp \left(-2.30259\left(\frac{|x+\Delta|}{w}\right)^{\frac{1.200545}{\ln (c)}}\right)
\end{array}\right.
$$

The total illumination distribution is

$$
F x(x, w, c, \Delta)=f x_{0}+f x_{1}+f x_{2}
$$

In many cases, two-dimensional global distribution (12) can be described by one-dimensional illumination distribution (14).

\section{Variation Rule of Illumination Distribution of Surgical Luminaires}

Using (13) and (14), it is easy to get the picture of variety of spots with spot radius $\mathrm{w}$ and spot shape $\mathrm{c}$ for surgical lamps.

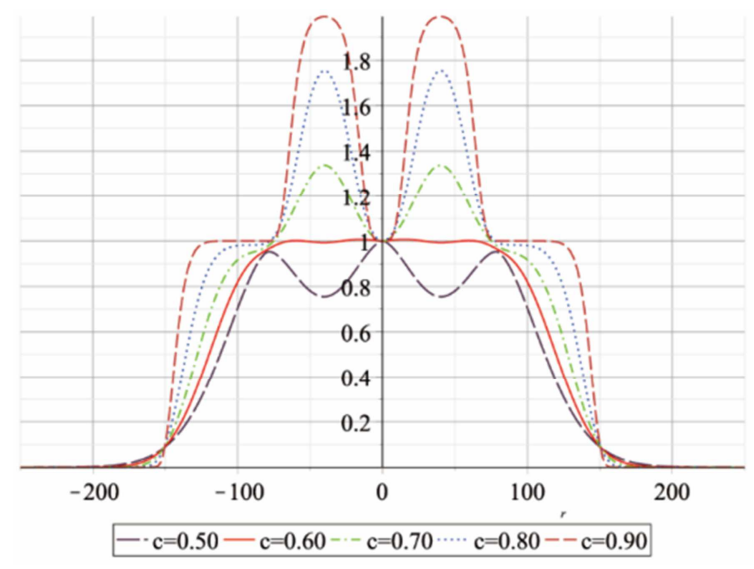

Figure 4. The overall illumination versus shape parameter $c$.

Firstly, the illuminate change with shape parameter $\mathrm{c}$ is analyzed. Figure 4 illustrates the relationship between total illuminance $\operatorname{Fx}(\mathrm{x}, \mathrm{w}, \mathrm{c}, \Delta)$ and $\mathrm{c}$ when $\mathrm{w}=70 \mathrm{~mm}$ and $\Delta=80$. The variation of $\mathrm{c}$ in the figure is within the range of $\mathrm{c}=0.5$ 0.9 which meets the IEC standard.

Take notice that in Figure 4 when $c=0.5$, there are two depressions. This is obvious because the "fullness" is not large enough. But unexpectedly, when the value of $\mathrm{c}$ is reach 0.7-0.9, there are two prominent peaks near $\mathrm{r}= \pm 45$. This is because of the degree of fullness is too large. Only when the value of $\mathrm{c}$ is about 0.6 , the illumination is relatively uniform. Similar results are obtained for other $\mathrm{w}$ and $\Delta$ values. The exact calculation shows that $\mathrm{c}=0.61$ is the best value in our design example.

This analysis finds that the spot is not the fuller the better, there is an optimum c value, which makes the spot have a better uniformity in all sizes. This is a valuable conclusion for surgical lamp designers.

Secondly, the variation of illumination distribution with total spot size was analyzed. It should be pointed out that it is not the size of the spot but the ratio of the maximum spot to the minimum spot that affects the uniformity of illumination. Of course, it is optimal if the total spot can change in a wide range, although a large range will inevitably lead to uneven spot. With the help of the analysis method in this paper, we can get a wide change range and keep a high uniformity. In our design example, the spot changes from $140 \mathrm{~mm}$ to $320 \mathrm{~mm}$, the change ratio is 2.3 times. Using (13) and (14), with $\mathrm{c}=0.61$, the normalized curves are obtained see Figure 1. It can be seen that the uniformity of illumination is above 0.9 in all ranges of variation. Although IEC does not stipulate the illumination uniformity, our result is consistent enough for practical application. 


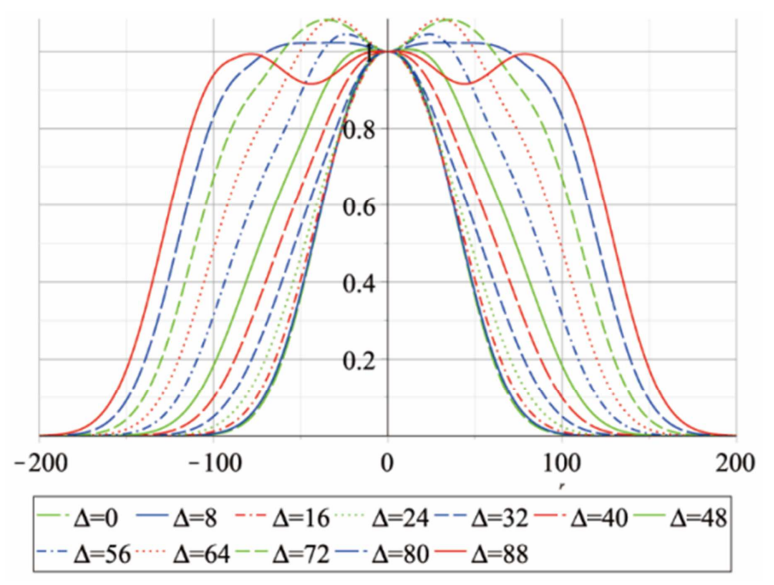

Figure 5. The overall illumination versus shift amount $\Delta$.

From above analysis, it can be determined $\mathrm{c}=0.61$ within the range $140-320 \mathrm{~mm}$, and the shape of the illumination distribution of the spot should be as follows:

$$
\exp \left(-0.0001(r)^{1.2144}\right)
$$

This form (15) is the basic requirement of the optical design of LED lens in this design. Specific lens design methods are not within the scope of this paper.

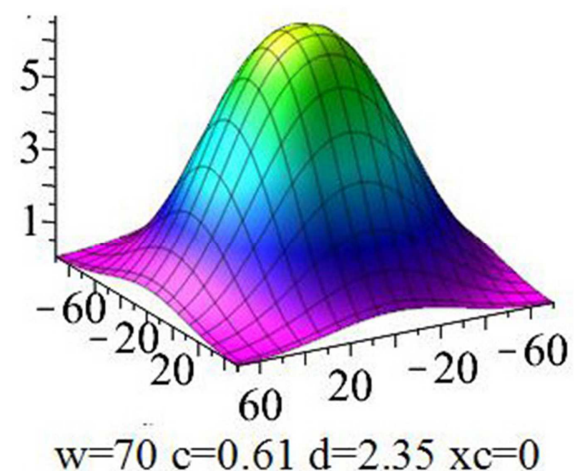

Figure 6. Minimum light field.

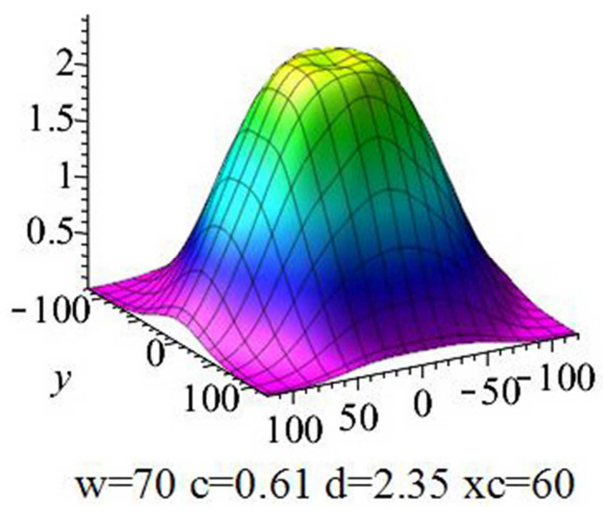

Figure 7. Medium size field.

Two-dimensional patterns can also be easily obtained by (11) and (12). Taking $\mathrm{c}=0.61$ for $\Delta=0,60$ and 90 respectively, the facula distribution Figure 6 Figure 7 and Figure 8 for the optimal lens are obtained.

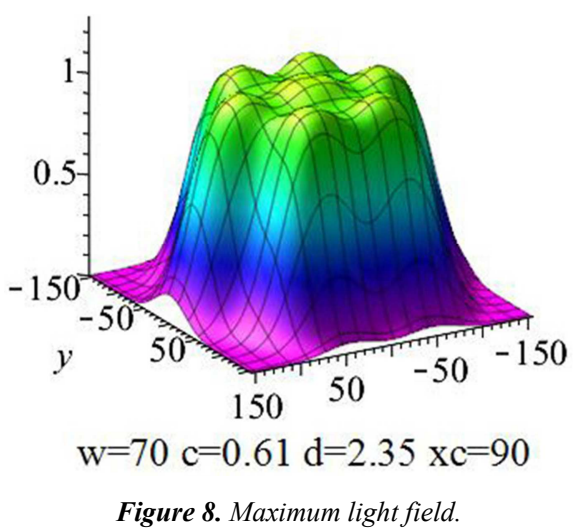

Shanghai Sansi has successfully manufactured the surgical lamp, which has passed the national test and can be mass produced. Figure 9 is the head part of model SSWYD800 lamp. Six modules change their elevation angle synchronously by mechanical transmission to move the beam spot of lighting area.

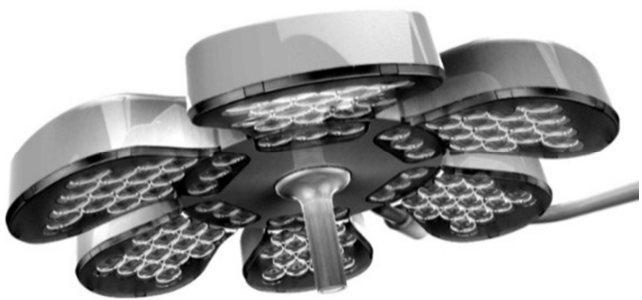

Figure 9. Surgical Luminaries model SSWYD-800.

\section{Discussions}

From the analysis in this paper, we find that the key quality index is not the size of shadow-less spot, but the "magnification" of spot change. Therefore, we think it is necessary to add a parameter of "optical magnification factor" for variable spot surgical lamp into IEC standard to characterize the performance of surgical lamps. It's just like the index "zoom multiple" in a zoom lens.

The example discussed in this paper has one fixed module and six peripheral active modules. In fact, this method can also be used in the design of more than seven modules of surgical lamps. It is assumed that the amplification factor can be increased by adding a circle of active modules outside the existing peripheral modules. We modeled a design of a lamp with 2 circles of modules, and the amplification factors can reach 4 at the same uniformity.

\section{Conclusions}

The modified mathematical model based on Gaussian function with multiple independent variables can be used to describe the illumination change of the LED surgical lamps for variable spot.

Under the condition of conforming to the IEC standard, for a certain "optical magnification factor" design, there is an optimum shape parameter "c", in which spot has the best uniformity in all sizes.

The surgical lamp constructed with seven LED modules can achieve the range 140-320 mm of spot changes, and 
meet the requirements of national standards and uniform illumination.

This method is suitable for the overall optical design of surgical lamps, including the single module intensity distribution, the peripheral module movement range and the total lighting spot variation range.

\section{References}

[1] IEC (International Electro-technical Commission) Standard 60601.2.41 2009 Particular requirements for basic safety and essential performance of surgical luminaires and luminaires for diagnosis Submission.

[2] National Pharmaceutical Industry Standards [S], YY06272008 .

[3] Knulst A J, Stassen L P S, Grimbergen C A, et al. "Standards and Performance Indicators for Surgical Luminaires" [J]. LEUKOS, 2009, 6 (1): 13.

[4] Forrester Jared A, Boyd Nicholas J, Fitzgerald J Edward F, et al. Impact of Surgical Lighting on Intraoperative Safety in Low-Resource Settings: A Cross-Sectional Survey of Surgical Providers. [J]. World Journal of Surgery, 2017, 41 (12): 3055-3065.

[5] Kamsah, Nazri \& Mohamed Kamar, Haslinda \& Alhamid, Muhammad \& Wong, Keng Yinn \& Akademia Baru, Penerbit. (2018). "Impacts of Temperature on Airborne Particles in A Hospital Operating Room", Journal of Advanced Research in Fluid Mechanics and Thermal Sciences. 44. 12-23.

[6] “Technical Guidance Document: LED Surgical Task Lighting” (PDF). Department of Energy. Retrieved 30 May 2012.

[7] JJ Mundingger and Kevin W. Houser, "Adjustable correlated colour temperature for surgical lighting" [J], Lighting Res. Technol. 2017; 0:1-11 Is it possible to help improve surgeries by mixing two LED lamps with four colour temperatures?

[8] Jingwei Liu, Yong Jiang and Jianjiu Li, "Development of a New Type of Medical LED Shadow-less Lamp for Surgery" [J], Semiconductor Lighting, 2010.6.

[9] Qiang Wang, "Design of LED Surgical Lamps", Huazhong University of Science and Technology [D], 2011

[10] Benhua Su, Chunying Su, Haiyan Yang, Nianlung Fen and
Zhongxuan Sun, "Development of Operating Shadow-less Lamp with LED Light Source" [J], Chinese Medical Equipment, 2017, 14 (11):1-5

[11] Meng Zhou, "Global Top Operative Lighting System" [J], China Medical Device Information, 2015,21 (02):68-69.

[12] Peng Liu, "Study on Freeform Surface LED Shadow-less Lamp with Adjustable Spectrum and Its Visual Perception" [D], Zhejiang University, 2014

[13] Shanghai Sansi Electronic Engineering Co. LTD, New Practical Patent: "Light spot adjustable LED shadow-less lamp" [P], Authorization Notice Number: CN 102661577 B (2013)

[14] Ide, Takeshi \& Kinugawa, Yoshitaka \& Nobae, Yuichi \& Suzuki, Toshihiro \& Tanaka, Yoshiyuki \& Toda, Ikuko \& Tsubota, Kazuo. (2015). "LED Light Characteristics for Surgical Shadowless Lamps and Surgical Loupes", Plastic and Reconstructive Surgery - Global Open. 3. 1. 10.1097/GOX. 0498.

[15] Pan, C. T. \& Chen, Candice \& Yang, T. L. \& Lin, Po-Hsun \& Lin, Po-Hung \& Huang, Jacob. (2015). "Study of reflectiontyped LED surgical shadowless lamp with thin film Ag-based metallic glass" [J], Optik - International Journal for Light and Electron Optics. 127. 10.1016/j.ijleo.2015.11.150.

[16] Zheng, Chaoqiang \& Dai, Min \& Zhang, Zhisheng \& Hu, Yubo. (2016). "Intelligent control system of medical shadowless lamps" based on STM32. 1-4. 10.1109/M2VIP.2016.7827327.

[17] Hajdau, Cornel \& Bistricianu, Maria. (2017). "Electric supply availability for the surgical luminaires", 426-429. 10.1109/EHB.2017.7995452.

[18] Shanghai Sansi Electronic Engineering Co. LTD, Invention Patent: "Overall Optical Design of LED Surgical Luminaires of Variable Light Spot" [P], Authorization Notice Number: CN 102661577 B (2017).

[19] Hongwei Guo, "A simple algorithm for fitting a Gaussian function" [J], IEEE Sign. Proc. Mag. 28 (9): 134-137 (2011).

[20] Oren Aharon, "Laser beam profiling and measurement" [J], Novus Light Technologies Today, (2013).

[21] Nathan Hagen, Matthew Kupinski, and Eustace L. Dereniak, "Gaussian profile estimation in one dimension" [J], Appl. Opt. 46, 5374-5383 (2007).

[22] N. Hagen and E. L. Dereniak, "Gaussian profile estimation in two dimensions" [J], Appl. Opt. 47:6842-6851 (2008). 